\title{
Experimental Results of a Differential Angle-of-Arrival Based 2D Localization Method Using Signals of Opportunity
}

\author{
M. A. Aziz ${ }^{1}{ }^{1}$ and C. T. Allen ${ }^{2}$ \\ ${ }^{1}$ Maury Microwave Corporation, Ontario, CA, USA \\ ${ }^{2}$ Electrical and Computer Engineering, University of Kansas, Lawrence, KS, USA \\ Correspondence should be addressed to M. A. Aziz; masudalaziz@outlook.com
}

Received 29 October 2017; Revised 11 February 2018; Accepted 20 February 2018; Published 25 March 2018

Academic Editor: Sandro M. Radicella

Copyright (C) 2018 M. A. Aziz and C. T. Allen. This is an open access article distributed under the Creative Commons Attribution License, which permits unrestricted use, distribution, and reproduction in any medium, provided the original work is properly cited.

\begin{abstract}
This paper presents a study of differential AoA (Angle-of-Arrival) based 2D localization method utilizing FM radio signals $(88 \mathrm{MHz}-108 \mathrm{MHz})$ as Signals of Opportunity (SOP). Given prior knowledge of the transmitters' position and signal characteristics, the proposed technique utilizes triangulation to localize receiver's 2D position. Dual antenna interferometry provides the received signals' AoA required for triangulation. Reliance on precise knowledge of antenna system's orientation is removed by utilizing differential Angle of Arrivals (dAoAs). The 2D localization accuracy is improved by utilizing colocated transmitters, a concept proposed in this paper as supertowers. Analysis, simulation, and ground-based experiments have been presented; results showed that when the SNR (Signal-to-Noise Ratio) is higher than $45 \mathrm{~dB}$, the proposed method localizes the receiver's $2 \mathrm{D}$ position with an error of less than $15 \mathrm{~m}$.
\end{abstract}

\section{Introduction}

Satellite based navigation system, such as GNSS (Global Navigation Satellite System), suffers from poor availability in challenging environments, such as indoor or urban area and is subject to detrimental interferences, such as jamming and spoofing [1]. Due to a plethora of ambient RF signals, a new paradigm, termed as OpNav (Opportunistic Navigation) using SOP (Signal of Opportunity), has been proposed to overcome the limitation of satellite based navigation system [2]. SOPs are signals that are transmitted for purposes other than navigation, such as AM/FM radio [3], cellular [4], digital television [5], iridium satellite [6], and Wi-Fi signals [7, 8].

Current trends in OpNav are based on trilateration (where timing of the transmitted signal is known a priori) and triangulation (where the antenna system's orientation is known a priori). A dAoA (differential Angle-of-Arrival) based localization system eliminates the aforementioned requirements where neither the timing of the transmitted signals nor the antenna system's orientation needs to be known a priori. Moreover, in AoA (Angle-of-Arrival) based localization system, the receiver needs to track an off-ground source accurately to eliminate the spurious effect caused by the phase difference in the plane defined by the line of sight and the line of separation of the apertures [9]. Therefore, this paper proposes a dAoA based 2D localization method utilizing FM radio signals as SOP. The choice of FM radio signals ( $88 \mathrm{MHz}-108 \mathrm{MHz}$ ) as SOP lies in the fact that the numbers of FM band transmitters are often higher in number than its SOP counterparts and FM transmitters for different FM stations (operating at different frequencies) are often colocated on the same tower and thus provides an opportunity to improve the localization accuracy by postprocessing AoAs of the colocated FM transmitters.

The contribution of this paper is twofold. First, it demonstrates a dAoA based 2D localization method that eliminates requirement of knowledge about antenna system's orientation. Second, it presents the experimental results supporting the claim that the localization accuracy can be improved by exploiting supertowers. In this paper, supertowers are termed as towers that host two or more FM transmitters operating at different frequencies.

The proposed concept is appropriate for applications, such as indoor (e.g., arenas, office spaces, warehouses, hospitals, hangers, and homes) and underground (e.g., caves, 


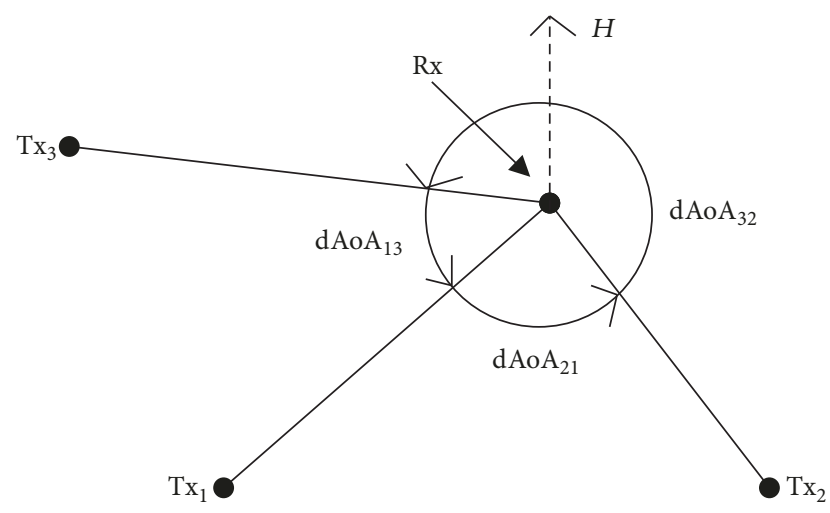

FIgURE 1: dAoAs between transmitter pairs.

mines, bunkers, and tunnels) 2D localization where signals from satellite are unavailable or received signal strength from satellite is low for accurate localization.

\section{Model Description}

The dAoA based triangulation is based on dAoAs between SOP transmitters (Tx) as shown in Figure 1.

In Figure $1, \mathrm{Tx}_{x}$ is the state vector of the SOP transmitter $x$ with $2 \mathrm{D}$ position states $\left(\mathrm{Tx}_{x} \triangleq\left[X_{x}, Y_{x}\right]^{T}\right), \mathrm{Rx}$ is the receiver state vector with $2 \mathrm{D}$ position states $\left(\mathrm{Rx} \triangleq[X, Y]^{T}\right)$, and $\mathrm{dAoA}_{x y}$ is the difference between $\mathrm{AoA}_{x}$ and $\mathrm{AoA}_{y}$ as shown in

$$
\mathrm{dAoA}_{x y}=\mathrm{AoA}_{x}-\mathrm{AoA}_{y}
$$

where $\mathrm{AoA}_{x}$ and $\mathrm{AoA}_{y}$ are the AoAs from transmitters $x$ and $y$, respectively. The AoAs can be measured exploiting a two antenna element interferometry as shown in

$$
\theta=\cos ^{-1}\left(\frac{\lambda}{2 \pi d} \varphi\right)
$$

where $\varphi$ is the phase difference between the received signals at two antenna elements, $d$ is the separation between antenna elements $(d \leq \lambda / 2$, where $\lambda$ is the wavelength at the operating frequency), and $\theta$ is the AoA of the corresponding SOP Tx relative to the interferometry baseline. With a set of at least 3 SOP Tx, a set of simultaneous equations can be constructed and the $2 \mathrm{D}$ localization of the receiver can be resolved.

\section{Higher Localization Accuracy Utilizing Supertowers}

FM band signals spread from $88 \mathrm{MHz}$ to $108 \mathrm{MHz}$ with $200 \mathrm{kHz}$ channel spacing. However, during the FFT of the received signals, a $10 \mathrm{kHz}$ frequency bin was found to be the optimum to maximize SNR and extract accurate phase information. This leads to a question of which frequency bin or bins are to be selected to measure accurate AoA and supertowers provide the answer on which frequency bins to choose.

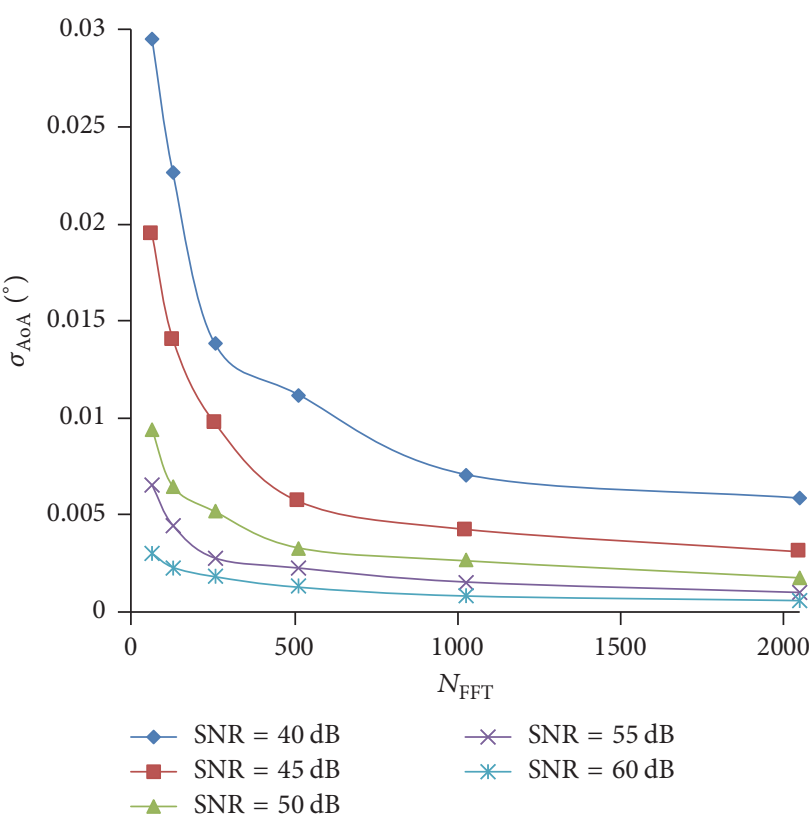

FIgURE 2: AoA accuracy versus $N_{\text {FFT }}$ versus SNR.

The phase differences between the received signals that are transmitted from two colocated FM transmitters (transmitting at different frequencies) should be related to each other as shown in

$$
\cos ^{-1}\left(\frac{\lambda_{1}}{2 \pi d} \varphi\right)=\cos ^{-1}\left(\frac{\lambda_{2}}{2 \pi d} \varphi^{\prime}\right),
$$

where $\varphi$ and $\varphi^{\prime}$ are the phase differences between the same received signals at two different sensors from colocated FM transmitters. It can be seen in (3) that the product of phase difference $(\varphi)$ and $\lambda / 2 \pi d$ should be equal to each other for colocated FM transmitters. This mathematical condition can then be applied to find the optimum frequency bin to calculate AoA accurately.

\section{Simulation}

The dAoA based 2D localization accuracy depends on the AoA accuracy of each SOP transmitter. The measured AoA of a Tx is a function of the number of samples during FFT $\left(N_{\mathrm{FFT}}\right)$, SNR (Signal-to-Noise Ratio) of the received signal at the receiver, and the spacing between $\mathrm{Rx}$ antenna elements. The received signal is assumed to have a thermal noise spectrum that is modelled by Gaussian Random sequence. The received signals at two antenna elements are then modelled by signal + noise contribution with a set of SNR and $N_{\mathrm{FFT}}$. The AoAs are then averaged over 1000 trials and the corresponding standard deviation of AoA error $\left(\sigma_{\mathrm{AoA}}\right)$ is shown in Figure 2.

It can be seen in Figure 2 that the AoA accuracy reaches its optimum value after 1024 samples. In order to find the impact of AoA error $\left(\sigma_{\mathrm{AoA}}\right)$ on $2 \mathrm{D}$ localization, a Gaussian Random sequence of 1024 samples has been modelled as $\sigma_{\text {AoA }}$ and 


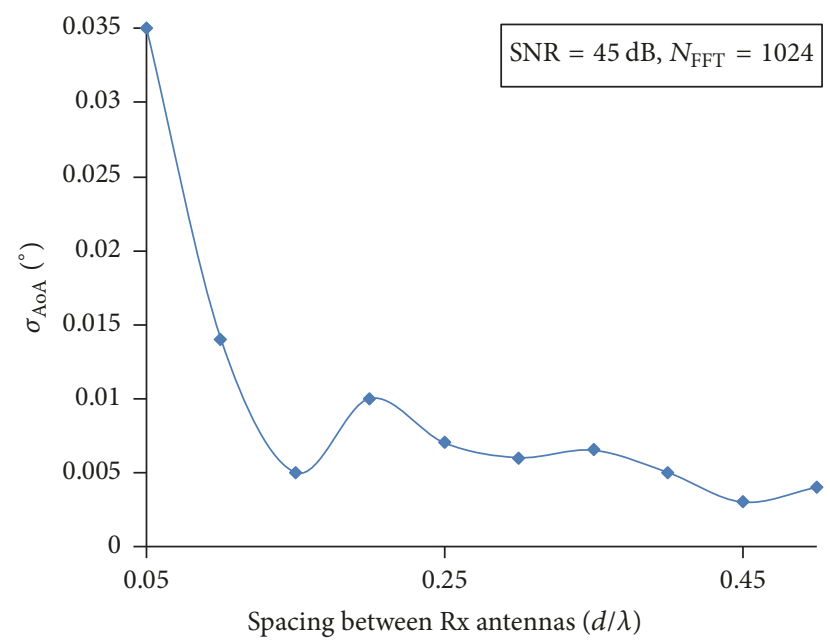

FIgURE 3: AoA accuracy versus spacing between $\mathrm{Rx}$ antenna elements.
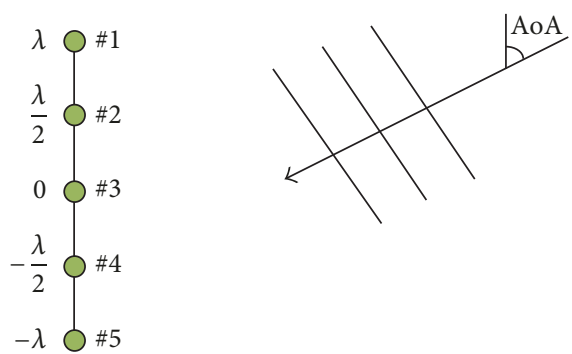

FIgURE 4: A uniformly spaced 5 elements (identical) Rx antenna array for measuring AoA.

TABLE 1: Impact of AoA accuracy over Rx localization.

\begin{tabular}{lc}
\hline$\left(\sigma_{\mathrm{AoA} 1}, \sigma_{\mathrm{AoA} 2}, \sigma_{\mathrm{AoA} 3}\right)($ degree $)$ & Localization error $(\mathrm{m})$ \\
\hline$(0.05,-0.05,0.05)$ & 119.5 \\
$(0.025,-0.025,0.025)$ & 71.8 \\
$(0.01,-0.01,0.01)$ & 23.9 \\
$(0.005,-0.005,0.005)$ & 11.9 \\
\hline
\end{tabular}

added to true AoA. The resulting AoAs are then triangulated to find the localization error and the result is shown in Table 1.

In this paper, the localization accuracy is targeted to be less than $15 \mathrm{~m}$ and thus a minimum SNR of $45 \mathrm{~dB}$ has been chosen based on Figure 2 and Table 1.

The effect of spacing between antenna elements was investigated while keeping $N_{\mathrm{FFT}}$ fixed to 1024 and SNR fixed to $45 \mathrm{~dB}$ as shown in Figure 3.

In this paper, the spacing between the $\mathrm{Rx}$ antennas was chosen to be $\lambda / 2$, where $\lambda$ is the free-space wavelength. The assumed values of all the parameters are shown in Table 2 .

The effect of number of Rx antenna elements on the AoA accuracy has also been studied. Consider 5 uniformly spaced antenna arrays with identical antenna elements as shown in Figure 4.

In Figure 4, the spacing between the antenna elements are chosen to be $\lambda / 2$ and Tx transmitters are considered to be in
TABLE 2: Assumed values of the parameters that affect AoA accuracy.

\begin{tabular}{lc}
\hline Parameter & Value \\
\hline$N_{\mathrm{FFT}}$ & 1024 \\
$\mathrm{SNR}$ & $45 \mathrm{~dB}$ \\
Separation between Rx antenna elements & $\frac{\lambda}{2}$ \\
\hline
\end{tabular}

TABLE 3: $\sigma_{\mathrm{AoA}}\left({ }^{\circ}\right)$ values for different $\mathrm{Rx}$ antenna combinations.

\begin{tabular}{lc}
\hline Antenna pair & $\sigma_{\mathrm{AoA}}\left(^{\circ}\right)$ \\
\hline 1,2 & 0.0033 \\
2,3 & 0.0028 \\
3,4 & 0.0035 \\
4,5 & 0.0032 \\
Average & 0.0032 \\
\hline
\end{tabular}

the far field of the Rx antennas and thus the receiving waves are plane waves. The AoAs are then calculated for each $\mathrm{Rx}$ pair with a separation of $\lambda / 2$ between them. Finally, all the AoAs from all the pairs are averaged to find out the effect of number of antenna elements as shown in Table 3.

It can be seen in Table 3 that the AoA accuracy from the 5 elements antenna array is similar to that obtained from a 2 elements antenna array interferometry. However, a 2-element antenna array introduces an AoA ambiguity between $(-\pi, 0)$ and $(0, \pi)$. Therefore, a third element is necessary to eliminate the AoA ambiguity. Thus, a 3-elements antenna array is the optimum size for accurate and ambiguity free interferometry.

\section{Minimum Clearance Condition between $T x$ and $R x$}

It is shown in [10] that the LoS (Line of Sight) calculation is based on earth's radius as well as the altitudes of the Tx and $\mathrm{Rx}$ as shown in

$$
R=\sqrt{2 K R_{\text {earth }}}\left(\sqrt{h_{1}}+\sqrt{h_{2}}\right),
$$

where $R$ is the total LoS, $R_{\text {earth }}$ is the radius of the earth, $h_{1}$ and $h_{2}$ are the Tx and the Rx antenna heights, respectively, and $k$ is the $k$-factor of the earth bulge. Under normal weather condition, $k=4 / 3$, whereas it becomes less than 1 in stormy weather to cause fading in transmission. Therefore, (4) can be expressed as in (5) to calculate LoS under normal weather condition.

$$
R=1.41\left(\sqrt{h_{1}}+\sqrt{h_{2}}\right)
$$

where $R$ is the total LoS in miles and $h_{1}$ and $h_{2}$ are in feet. For example, with a Tx antenna altitude of 600 feet (typical of an FM tower) and a Rx antenna altitude of 400 feet (representative of the altitude ceiling for a small UAV under current FAA rules), the maximum range can be estimated from (5) to be 62.73 miles or $100 \mathrm{~km}$. On the other hand, with the same Tx antenna height but with a 6 feet $\mathrm{Rx}$ antenna height (typical of a ground-based measurement), 


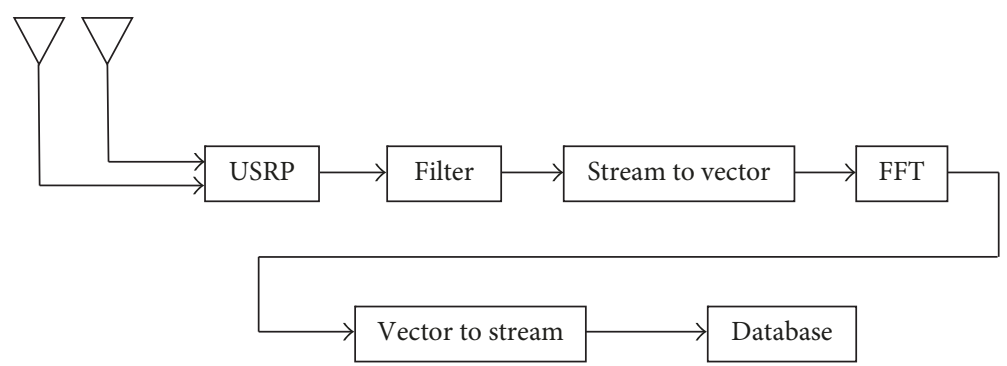

FIgURe 5: A generic GRC flow graph.

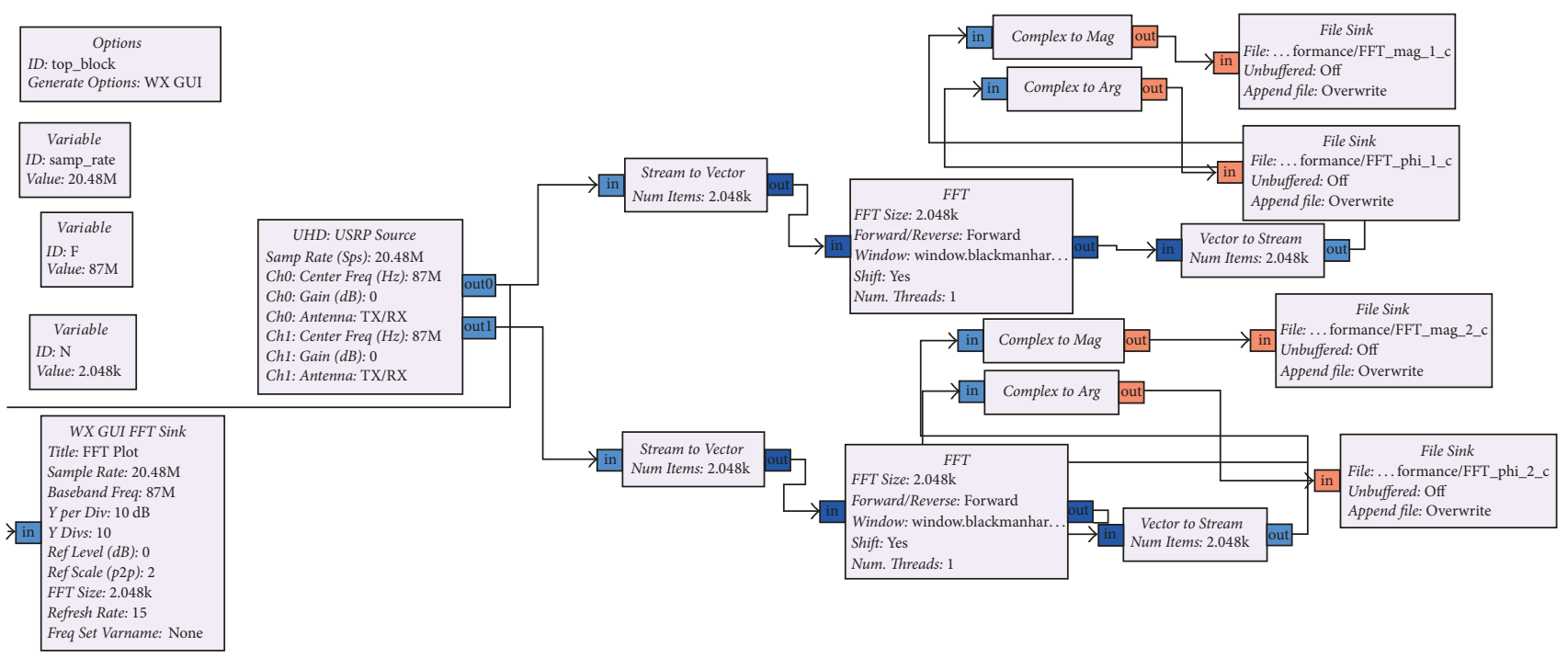

FIGURE 6: GRC flow graph for SDR card to receive two signals at two ports.

the maximum range shrinks to $60 \mathrm{~km}$. Since the proposed localization method is demonstrated based on FM transmitters and ground-based measurement, the maximum range is estimated to be $60 \mathrm{~km}$ for this paper.

\section{Measurement Setup}

The proposed method has been validated by performing measurement utilizing a dual channel receiver using SDR (Software Defined Radio: model B210) [11]. The B210 SDR card contains $2 \mathrm{Tx}$ and $2 \mathrm{Rx}$ ports with fully coherent $2 \times 2$ MIMO (Multiple Input Multiple Output) capabilities. The coherency between the Rx ports is an essential feature for extracting accurate phase difference between the received signals. Moreover, the SDR card covers RF frequencies from $70 \mathrm{MHz}$ to $6 \mathrm{GHz}$ with a bandwidth of maximum $30.72 \mathrm{MHz}$ in $2 \times 2$ MIMO modes. Thus the B210 SDR card was an ideal candidate for this project. Besides, the B210 board comes with an AD9361 chip that performs the function of ADC and DSP.

The receiver utilizes 2-element antenna array interferometry to extract the phase difference between its 2 channels. The measured phase difference is then utilized to calculate the AoAs, dAoAs, and 2D position estimate. The SDR card was configured with the GNU Radio Companion (GRC) interface. GRC is a graphical user interface to build GNU radio flow graphs or the software circuits. The GNU radio flow graph enables the SDR card to receive signals from the two antennas, spectrally isolate them, and then calculate the phase difference between the received signals. A generic block diagram of GRC flow graph is shown in Figure 5.

A description of all the blocks can be found in [12]. A sample implementation of each block in Figure 5 is shown in Figure 6.

A picture of the complete measurement setup is shown in Figure 7.

\section{Experimental Results}

Ground-based experiment was conducted at Blue Valley Park in Kansas City, MO in USA. The serving FM band Tx locations were found in FCC database [13] as shown in Table 4.

The SNR of all the frequency bins for all the Tx frequencies at tower 2 is shown in Figure 8(a). Moreover, the products of phase differences and $\lambda / 2 \pi d$ are shown in Figure $8(\mathrm{~b})$.

The selection of the appropriate frequency bin depends on two factors, such as the SNR $>45 \mathrm{~dB}$ (Figure 8(a)) and the product terms being close to each other. Based on Figures 8(a) and 8 (b), the optimum frequency bins for tower 2 are found as shown in Table 5. 


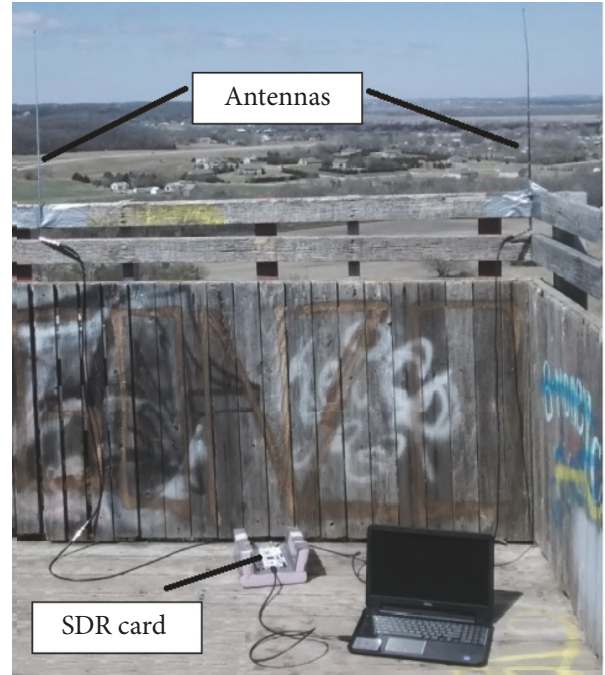

FIGURE 7: A picture of the complete measurement setup.

TABLE 4: The colocated FM transmitters.

\begin{tabular}{lcccc}
\hline Tower index & \multicolumn{4}{c}{ Tx frequency $(\mathrm{MHz})$} \\
\hline 1 & 88.5 & - & - & - \\
2 & 93.3 & 103.3 & 94.1 & - \\
3 & 89.3 & 104.3 & - & - \\
4 & 94.9 & 102.1 & 105.1 & 97.3 \\
5 & 90.1 & - & - & - \\
\hline
\end{tabular}

TABLE 5: Calculated AoAs from optimum frequency bins for tower 2.

\begin{tabular}{lccc}
\hline Tx freq. (MHz) & Calc. AoA $\left({ }^{\circ}\right)$ & Optimum bin & Highest SNR bin \\
\hline 93.3 & -28.137 & 11 & 11 \\
103.3 & -28.42 & 12 & 12 \\
94.1 & -28.48 & 15 & 14
\end{tabular}

The AoA of tower 2 is then calculated to be the average of all 3 AoAs. It should be noted here that the optimum frequency bin is not the frequency bin with the highest SNR. A similar algorithm was applied to towers 3 and 4 to calculate the corresponding AoAs.

The calculated AoAs are then triangulated to localize the receiver and the Rx position error is calculated as shown in

$$
\begin{aligned}
& \mathcal{E}_{\text {pos }} \\
& =\sqrt{\left(\mathrm{Rx}_{x}(\text { true })-\mathrm{Rx}_{x}(\text { calc })\right)^{2}+\left(\mathrm{Rx}_{y}(\text { true })-\mathrm{Rx}_{y}(\text { calc })\right)^{2}},
\end{aligned}
$$

where $\left(\mathrm{Rx}_{x}\right.$ (true), $\mathrm{Rx}_{y}($ true $\left.)\right)$ are the true $\mathrm{Rx}$ positions, $\left(\mathrm{Rx}_{x}(\mathrm{calc}), \mathrm{Rx}_{y}(\mathrm{calc})\right)$ are the calculated $\mathrm{Rx}$ positions, and $\varepsilon_{\text {pos }}$ is the localization error in meters. The resultant localization error for with and without supertowers are shown in Table 6 .

It can be seen from Table 6 that the localization accuracy is finest when utilizing all 3 supertowers.

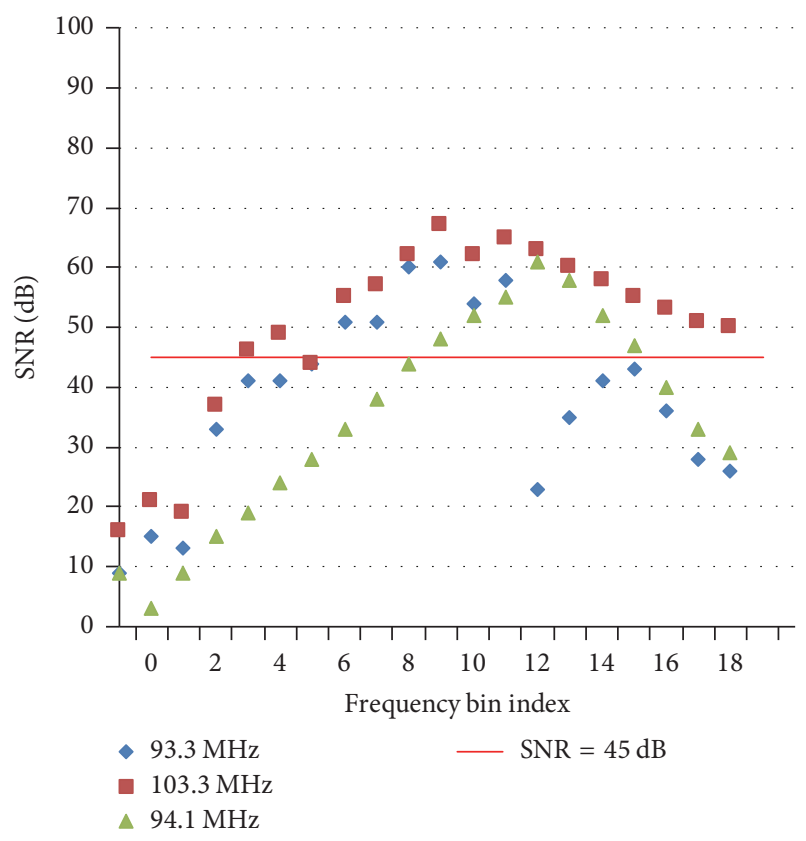

(a)

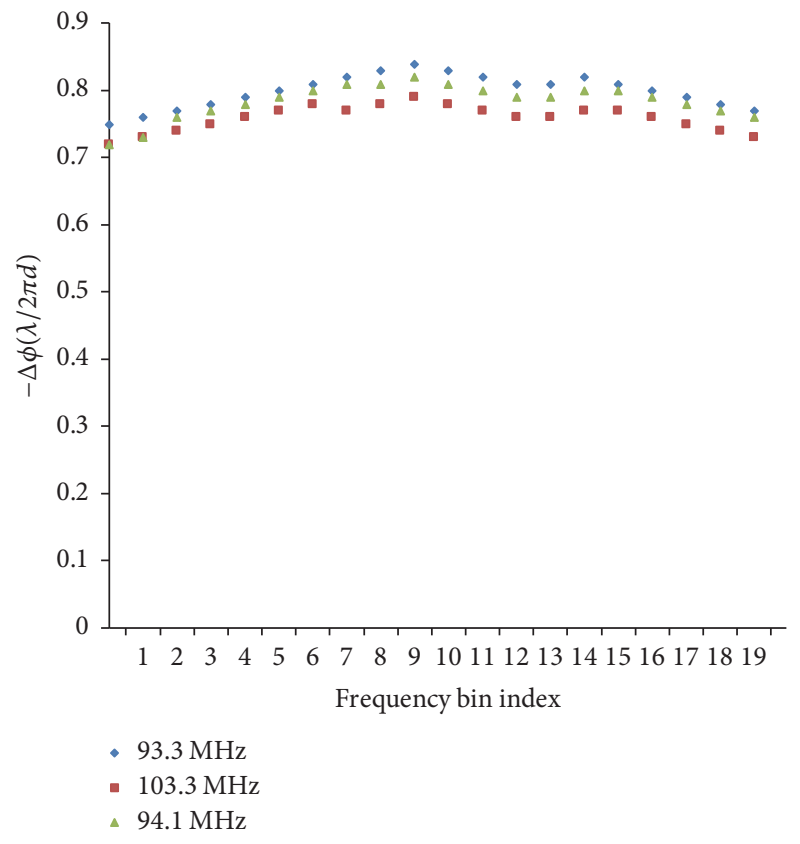

(b)

Figure 8: (a) Phase difference between received signals from tower 2. (b) Product of $\varphi$ and $\lambda / 2 \pi d$ for all transmitted signals from tower 2 .

\section{Conclusion}

In this paper, a dAoA based 2D localization method has been demonstrated using FM radio signals as SOP. A path to utilize colocated FM transmitters to obtain further localization accuracy has been proposed and validated through experimental results. It has been shown that the utilization of all supertowers provides the least error of $13 \mathrm{~m}$ in $2 \mathrm{D}$ localization. 
TABLE 6: A comparison of localization with and without supertowers.

\begin{tabular}{lcc}
\hline Number of used supertowers & Indices of the used supertowers & Localization error, $\varepsilon_{\text {pos }}$ (m) \\
\hline 3 & 2,3 , and 4 & 12.57 \\
2 & 1,2 , and 3 & 32.55 \\
2 & 1,2 , and 4 & 22.43 \\
2 & 1,3 , and 4 & 46.37 \\
2 & 2,3 , and 5 & 33.43 \\
2 & 2,4 , and 5 & 30.37 \\
2 & 3,4 , and 5 & 48.28 \\
1 & 1,2 , and 5 & 26.35 \\
1 & 1,3 , and 5 & 63.31 \\
1 & 1,4 , and 5 & 30.48 \\
\hline
\end{tabular}

\section{Conflicts of Interest}

The authors declare that there are no conflicts of interest regarding the publication of this paper.

\section{References}

[1] S. D'Addio and M. Martin-Neira, "Comparison of processing techniques for remote sensing of earth-exploiting reflected radio-navigation signals," IEEE Electronics Letters, vol. 49, no. 4, pp. 297-298, 2013.

[2] L. A. Merry, R. M. Faragher, and S. Scheding, "Comparison of Opportunistic Signals for Localisation," in Proceedings of the 7th IFAC Symposium on Intelligent Autonomous Vehicles, vol. 43, pp. 109-114, 2010.

[3] V. Moghtadaiee and A. G. Dempster, "Indoor location fingerprinting using FM radio signals," IEEE Transactions on Broadcasting, vol. 60, no. 2, pp. 336-346, 2014.

[4] C. Yang, T. Nguyen, and E. Blasch, "Mobile positioning via fusion of mixed signals of opportunity," IEEE Aerospace and Electronic Systems Magazine, vol. 29, no. 4, pp. 34-46, 2014.

[5] M. Rabinowitz and J. J. Spilker Jr., "A new positioning system using television synchronization signals," IEEE Transactions on Broadcasting, vol. 51, no. 1, pp. 51-61, 2005.

[6] M. Joerger, L. Gratton, B. Pervan, and C. E. Cohen, "Analysis of Iridium-augmented GPS for floating carrier phase positioning," NAVIGATION: Journal of the Institute of Navigation, vol. 57, no. 2, pp. 137-160, 2010.

[7] I. Bisio, M. Cerruti, F. Lavagetto et al., "A trainingless WiFi fingerprint positioning approach over mobile devices," IEEE Antennas and Wireless Propagation Letters, vol. 13, pp. 832-835, 2014.

[8] Z. M. Kassas and T. E. Humphreys, "Receding horizon trajectory optimization in opportunistic navigation environments," IEEE Transactions on Aerospace and Electronic Systems, vol. 51, no. 2, pp. 866-877, 2015.

[9] D. L. Fried, "Differential angle of arrival: Theory, evaluation, and measurement feasibility," Radio Science, vol. 10, no. 1, pp. 71-76, 1975.

[10] C. Haslett, Essentials of Radio Wave Propagation, Cambridge University Press, 2008.

[11] Ettus Research, USRP B210 (Board Only), http://www.ettus .com/content/files/b200-b210specsheet.pdf.
[12] M. A. Aziz, Navigation for UAVs using Signals of Opportunity [Ph.D. thesis], Dept. of Electrical Engineering, University of Kansas, USA, 2015.

[13] http://www.zipsignal.v-soft.com, 2015. 


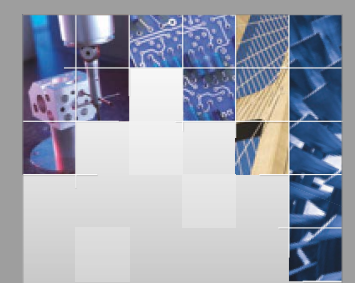

\section{Enfincering}
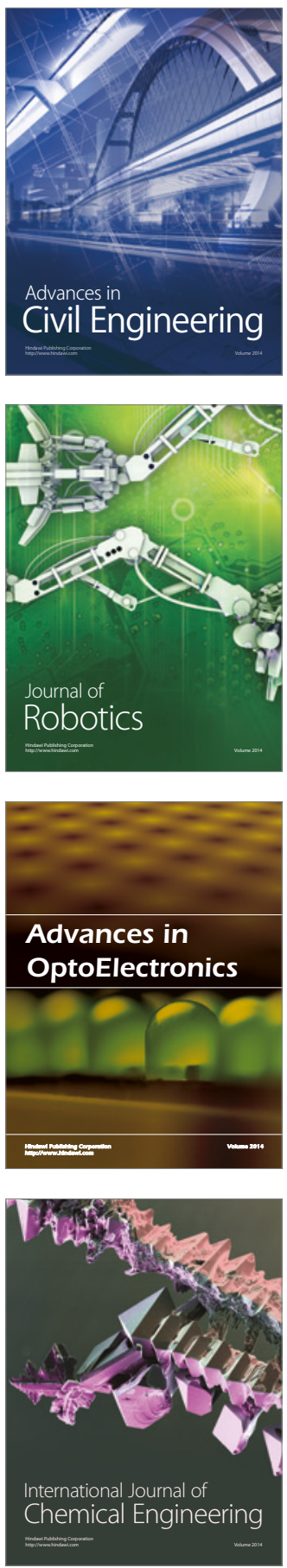

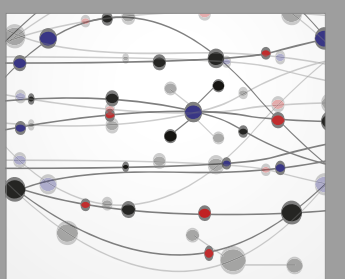

The Scientific World Journal
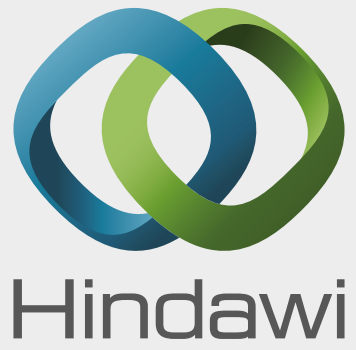

Submit your manuscripts at

https://www.hindawi.com
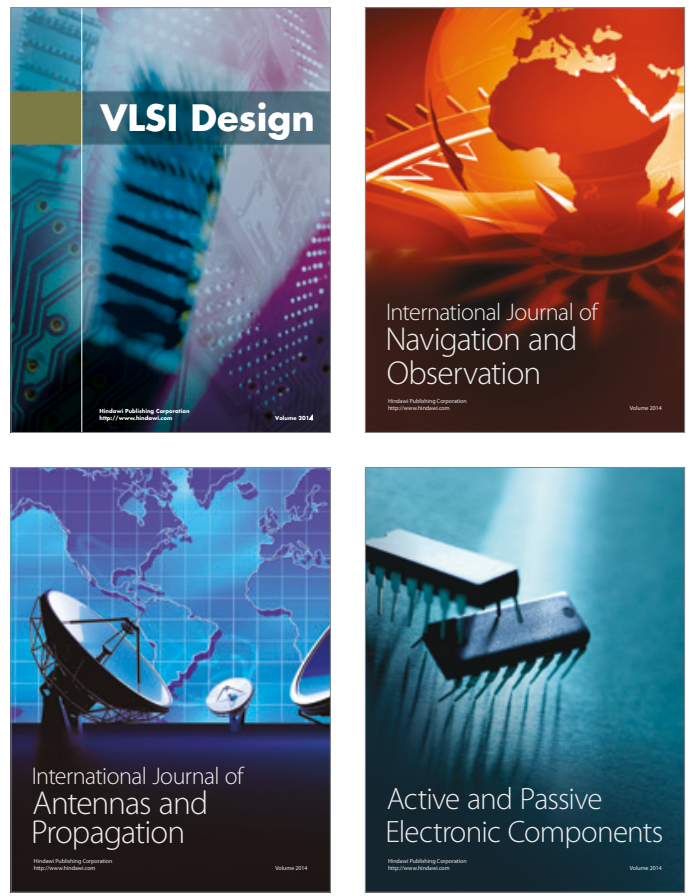

\section{Rotating}

Machinery

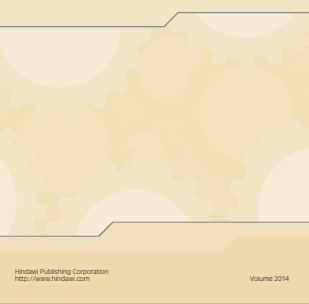

Journal of

Sensors

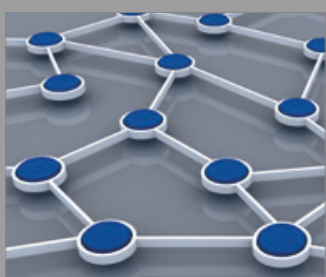

International Journal of

Distributed

Sensor Networks

Journal of

Control Science

and Engineering
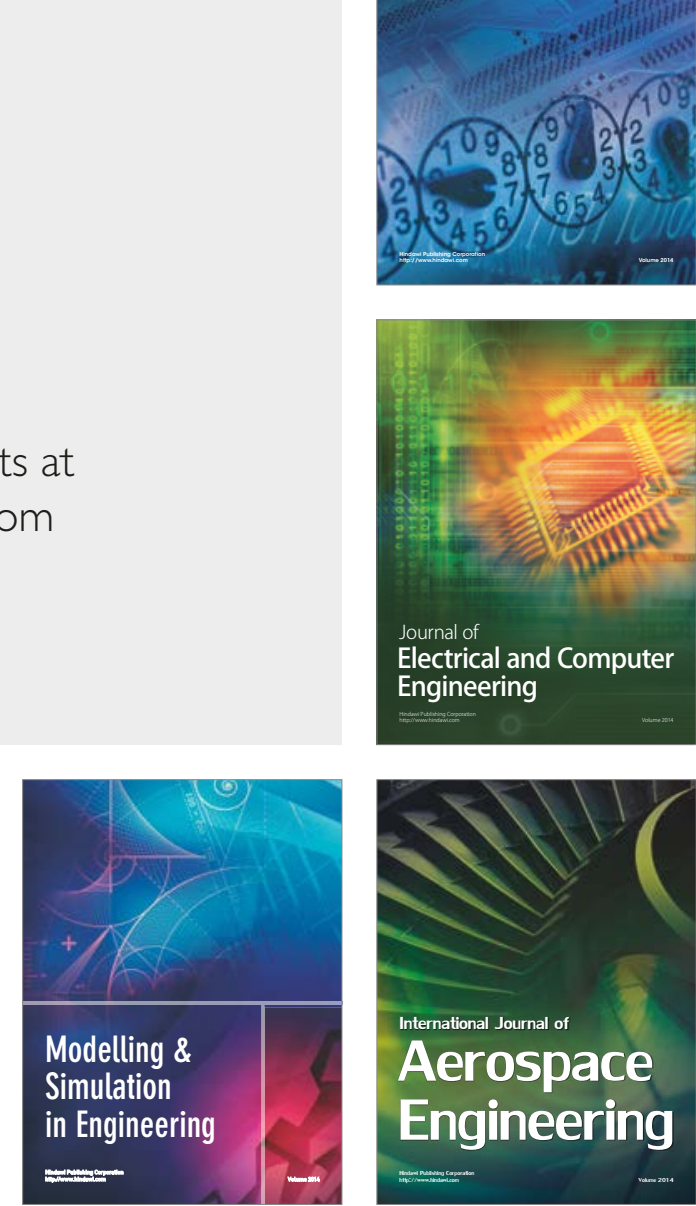
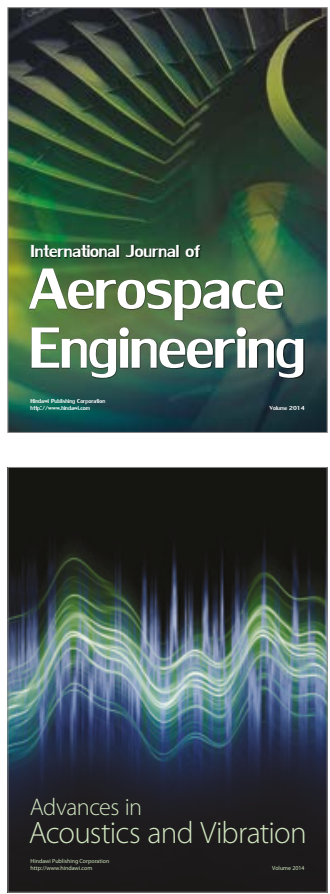